\title{
Knockdown of the nucleosome binding protein 1 inhibits the growth and invasion of clear cell renal cell carcinoma cells in vitro and in vivo
}

\author{
Shi-Qi Ji ${ }^{1,2,3}$, Lin Yao ${ }^{1,2,3}$, Xiao-Yu Zhang ${ }^{1,2,3}$, Xue-Song Li ${ }^{1,2,3}$ and Li-Qun Zhou ${ }^{1,2,3,4^{*}}$
}

\begin{abstract}
Background: The nucleosome binding protein 1 (HMGN5/NSBP1) is a member of the HMGN protein family and is highly expressed in several kinds of cancer. Nevertheless, the role of NSBP1 in clear cell renal cell carcinoma (cCRCC) remains unclear. This study aimed to confirm the oncogenic role of NSBP1 in cCRCC using in vitro and in vivo models and explore the mechanism by which NSBP1 contributes to $c C R C C$ tumorigenesis.

Methods: NSBP1 expression was detected in renal tissues from 152 ccRCC patients by immunohistochemistry, and examined in ccRCC cell lines by RT-PCR and Western blot analysis. cCRCC cells were transfected by NSBP1 RNAi and cell viability, apoptosis and invasion were detected by cell vitality test, flow cytometry and transwell assay in vitro. Xenograft in nude mice was also employed to examine the tumorigenesis of cCRCC cells depleted of NSBP1.

Results: Immunohistostaining showed strong immunoreactivity of NSBP1 in all cCRCC tissues and NSBP1 expression level was associated with tumor grade $(p=0.04)$. NSBP1 expression at mRNA and protein levels was high in cCRCC cell lines. Knockdown of NSBP1 induced cell cycle arrest and apoptosis, and inhibited invasion in 786-O cells. Western blot analysis demonstrated increased expression of Bax and decreased expression of Bcl-2, CyclinB1, VEGF, VEGFR-2, MMP-2, MMP-9, c-fos and c-jun in 786-O cells depleted of NSBP1. In vivo study further showed that knockdown of NSBP1 affected the tumorigenesis of ccRCC cells in nude mice.
\end{abstract}

Conclusions: NSBP1 plays oncogenic role in cCRCCs by promoting cell proliferation and invasion, and could be exploited as a target for $\mathrm{CCRCC}$ treatment.

Keywords: Clear cell renal cell carcinoma, NSBP1, Apoptosis, Cell cycle, MMPs

\section{Introduction}

Renal carcinoma is the 13th most common cancer worldwide, with clear cell and clear cell renal cell carcinoma (ccRCC) accounting for most of the renal cell carcinoma (RCC) [1]. Radical nephrectomy is effective to cure early and local ccRCCs, but advanced or metastatic ccRCCs barely respond to chemotherapy or radiotherapy and have poor prognosis. Therefore, it is important to better understand the pathogenesis of aggressive RCC in order to develop effective strategies for the prevention and treatment of RCC.

\footnotetext{
* Correspondence: zhoulqmail@china.com

'Department of Urology, Peking University First Hospital, Beijing 100034, China

Full list of author information is available at the end of the article
}

NSBP1 is a new member of the high mobility group $\mathrm{N}$ (HMGN) protein family that modulates the structure and function of chromatin and plays an important role in transcription, histone modifications, DNA replication and DNA repair in living cells[2]. Early study showed that nucleosome binding protein 1 (HMGN5/NSBP1) was abundantly expressed in prostate cancer [3]. In addition, NSBP1 expression was upregulated in squamous cell carcinoma, metastatic MDA-MB-435HM breast cancer cell line and adenocarcinoma, suggesting that NSBP1 may promote tumorigenesis [4-7].

Our previous studies showed that downregulation of NSBP1 expression caused G2 cell cycle arrest, decreased proliferation rate and increased apoptosis rate in prostate cancer cells in vitro $[8,9]$. Nevertheless, the role of NSBP1 in ccRCC development remains unknown.

\section{C) Biomed Central}


Tumor invasion and metastasis are complicated processes, among which proteolytic degradation of extracellular matrix (ECM) and angiogenesis (VEGF) are essential steps. ECM degradation can be promoted by the imbalance between proteolytic proteases and their inhibitors. Extensive studies have shown that matrix metalloproteinases (MMPs) play crucial role in the degradation of ECM to promote tumor invasion and metastasis $[10,11]$.

Therefore, in this study we investigated the role of NSBP1 in ccRCC. First we detected NSBP1 expression in clinical ccRCC tissues and ccRCC cell lines. Then we examined the effects of lentivirus mediated NSBP1 knockdown on the growth and invasion of ccRCC 786$\mathrm{O}$ cells and xenograft tumor growth in nude mice. The results showed that NSBP1 expression was upregulated in ccRCC tissues and ccRCC cell lines, and NSBP1 knockdown could induce apoptosis and inhibit the proliferation and invasion of ccRCC cells, and further decrease ccRCC tumor growth in nude mice.

\section{Methods}

\section{Clinical samples}

A total of 152 patients (aged 52 to 90 years old, median age of 64 years) who underwent surgery from January 2008 to January 2011 in Peking University First Hospital were enrolled in the present study. All patients were of Chinese origin. Paraffin wax-embedded blocks of tumor tissues from each patient were assembled from the archival collections at the Department of Pathology. Survival data of all patients were collected. Among these patients, 20 patients were randomly selected and paired cancer and adjacent tissues were collected from them for Western blot analysis of NSBP1 expression. All adjacent tissues were confirmed to be normal by experienced pathologists. The protocols for the present study were approved by the Ethics Committee of Peking University First Hospital.

\section{Cell culture}

The ccRCC cell lines Caki-2, A498, 786-O and the normal renal tubular epithelial line HK-2 were purchased from American Type Culture Collection (ATCC, Manassas, VA). HK-2 cells were cultured in K-SFM medium (Gibco $^{\mathrm{TM}}$ Life Technologies, Grand Island, NY), and other cells were cultured in RPIM-1640 (HyClone, Logan, UT) medium supplemented with $10 \%$ Gibco $^{\mathrm{TM}}$ FBS (Life Technologies, Grand Island, NY). All cells were cultured at $37^{\circ} \mathrm{C}$ in a standard humidified incubator containing $5 \% \mathrm{CO}_{2}$ and $95 \% \mathrm{O}_{2}$.

\section{Lentivirus RNAi construct and transfection}

The siRNA targeting the human NSBP1 (NM_030763) transcript was designed using the software developed by
Ambion (Foster, CA, USA) with the following sequence: PscSI616 CACAGCCTTTCTTTAGCATTTCAAGAGAATGCTAAAGAAAGG-CTGTG/CACAGCCTTTC TTTAGCATTCTCTTGAAATGCTAAAGA-AAGGC TGTG. NSBP1 siRNA or control scramble siRNA was cloned into vector. 786-O cells were seeded onto 6-well plates and grown to $60 \%$ confluence on the day of transfection. $4 \mathrm{~h}$ before transfection, cells were placed in serum-free media. Cells were transfected with $100 \mathrm{nM}$ siRNA vector diluted in RPMI-1640 according to the manufacturer's protocol. Successful knockdown of NSBP1 was analyzed by Western blot analysis and realtime PCR.

\section{Immunohistochemistry}

Paraffin-embedded tissues were cut into 4 um-thick consecutive sections and were then dewaxed in xylene and rehydrated in graded ethanol solutions. Antigen retrieval was performed following the standard procedure. Sections were cooled and immersed in a $0.3 \%$ hydrogen peroxide solution for 15 min to block endogenous peroxidase activity, and then rinsed in PBS for 5 min. Non-specific labeling was blocked by incubation with $5 \%$ bovine serum albumin at room temperature for $30 \mathrm{~min}$. Sections were then incubated with primary rabbit anti-human antibody against NSBP1 (diluted in 1:100, Abcam, ab56031, Cambridge, MA) at $4{ }^{\circ} \mathrm{C}$ overnight, rinsed with $\mathrm{PBST}$, incubated with horseradish peroxidase-conjugated Santa $\mathrm{Cruz}^{\mathrm{TM}}$ goat anti-rabbit IgG secondary antibody (Santa Cruz, CA), developed by peroxidase-conjugated streptavidin and $\mathrm{DAB}$, and counterstained by hematoxylin. All slides were examined independently by two pathologists, who were not informed about patients' clinical data. Specimens were then grouped according to stage (T1-T4) and specific staining intensity. The staining intensity was scored as "-" for negative, "+" for moderate, and "++" for strong staining.

\section{Quantitative real-time PCR assay}

Total RNA was extracted from the cells using Trizol (Invitrogen) according to the manufacturer's protocol. First-strand cDNA was generated using $2 \mu \mathrm{g}$ total RNA via MMLV-reverse transcriptase using High Capacity RNA-to-cDNA kit (Promega) with random primers. A final reaction of $20 \mathrm{ul}$ was used to determine the mRNA level by real-time PCR using an ABI Prism 7300 (Applied Biosystems, Foster City, CA, USA). The specific primers were as follows: NSBP1, 5'-TCGGCT TTTTTTCTGCTGACTAA-3'(forward) and 5'-CTC TTTGGCTCCTGCCTCAT-3'(reverse); Actin, 5'-GTG GACATCCGCAAAGAC3'(forward) and 5'-ATCAACGCAATGTGGGAAA-3'(reverse). Thermal cycling was initiated with a denaturation step for $5 \mathrm{~min}$ at $94^{\circ} \mathrm{C}$ 
followed by 36 cycles done in three steps: $30 \mathrm{~s}$ at $94^{\circ} \mathrm{C}$, $30 \mathrm{~s}$ at $58^{\circ} \mathrm{C}$ and $1 \mathrm{~min}$ at $72^{\circ} \mathrm{C}$.

\section{Cell proliferation assay}

Cell proliferation was assessed using the CellTiter 96 Aqueous assay kit (Promega, Madison, WI). After transfection, the cells $(10,000 /$ well $)$ were seeded in 96-well plates and incubated at $37^{\circ} \mathrm{C}$, and cell proliferation was assessed after $96 \mathrm{~h}$ based on the absorbance measured at $570 \mathrm{~nm}$ using a multiwell spectrophotometer.

\section{Flow cytometry}

Apoptosis was evaluated by Annexin V-PE/7-AAD staining followed by flow cytometry analysis. After cells were plated in 6-well plates at a density of $1 \times 10^{5} /$ well and cultured at $37^{\circ} \mathrm{C}$ in $5 \% \mathrm{CO} 2$ incubator for three days, they were transfected with NSBP1 siRNA or scramble siRNA vector, the cells were gently trypsinized and washed with ice-cold PBS after $72 \mathrm{~h}$. At least 20,000 cells were resuspended in $500 \mu \mathrm{L} 1 \times$ binding buffer, stained with $5 \mu \mathrm{L} 7$-AAD $\left(25 \mu \mathrm{g} \mathrm{mL}{ }^{-1}\right)$ and $1 \mu \mathrm{L}$ Annexin V-PE and immediately analyzed with a FACScalibur flow cytometer (Becton Dickinson, Erembodegem, Belgium).

\section{Western blot analysis}

inhibitors. Protein samples(40 ug)were separated in 10\% SDS-polyacrylamide gels and transferred to PVDF membranes. The membranes were blocked with nonfat milk in TBST, and probed with primary antibodies CyclinB1 (CST-4138), CyclinD1 (CST-2978), Proliferating Cell Nuclear Antigen (PCNA, CST-2586), Bax (CST-2772), Bcl-2 (CST-2876), VEGF (CST-2445), VEGFR-2 (CST2472), MMP-2 (CST-4022), MMP-9 (CST-3852) (CST indicated Cell Signaling Technology, Beverly, MA, USA). c-fos (santa cruz-52), c-jun (santa cruz-1694), GAPDH (santa cruz-137179), or $\beta$-Actin (santa cruz-81178), and secondary antibodies goat anti-mouse IgG (santa cruz), goat anti-rabbit IgG (santa cruz) (santa cruz indicated Santa Cruz Biotech, Santa Cruz, CA, USA). Immunoreactivity signals were developed using ECL kit (GE Healthcare Bioscience, Piscataway, NJ, USA). protease and phosphatase with Whole-cell extracts were prepared in RIPA buffer

\section{Cell invasion assay}

Cell invasion assay was performed with 24-well Transwell insert (pore size $8 \mu \mathrm{m}$, Corning, NY). After transfection, 786-O cells were starved in serum free medium overnight, and 3-5 $\times 10^{4}$ cells were resuspended in 200 ul serum-free medium and placed in the upper chambers with $8 \mu \mathrm{m}$ filter pores in triplicate. The membrane undersurface was coated with 30 ul ECM gel from Engelbreth-Holm-Swarm mouse sarcoma

(BD
Biosciences, Bedford, MA, USA) mixed with RPMI-1640 serum free medium in 1:5 dilution for $30 \mathrm{~min}$ at $37^{\circ}$. The lower chamber was filled with 500 ul $10 \%$ FBS as the chemoattractant and incubated for $48 \mathrm{~h}$. At the end of the experiments, the cells on the upper surface of the membrane were removed by cotton buds, and the cells on the lower surfaPBS-buffered paraformaldehyde and stained with $0.1 \%$ crystal violet. Five visual fields were chosen randomly for each insert and photographed under a light microscope at $200 \times$ magnification. The cells were counted and the data were summarized by means \pm standard deviation and presented by a percentage of controls. ce of the insert were fixed in $4 \%$.

\section{Gelatin zymography assay}

After transfection, the cells were cultured in serum free medium for $24 \mathrm{~h}$. Then the medium was collected by centrifugation at $4,000 \mathrm{rpm}$ for $15 \mathrm{~min}$ at $4^{\circ} \mathrm{C}$, and subjected to zymographic SDS-PAGE containing 0.1\% gelatin $(\mathrm{w} / \mathrm{v})$. The gels were washed and incubated in incubation buffer for $48 \mathrm{~h}$, then stained with Coomassie Brilliant Blue and destained. The zones of gelatinolytic activity were shown by negative staining.

\section{Tumourigenesis assay in nude mice}

Female BALB/ $\mathrm{c}^{\mathrm{nu} / \mathrm{nu}}$ mice (4-6 weeks old, weighed 25-30 g) were maintained in a germ-free environment in the animal facility. NSBP1 knockdown or control 786-O cells were cultured in 100-mm dishes and trypsinized. The cells $\left(10^{6}\right.$ in $100 \mathrm{ul} \mathrm{medium)} \mathrm{were} \mathrm{infused} \mathrm{subcutaneously} \mathrm{in} \mathrm{the}$ armpit area. Tumor diameter was measured every 5 days, and tumor volume was calculated by length $\times$ width $^{2} \times 0.5$. Mice were sacrificed after 1.5 months.

\section{Statistical analysis}

Values were represented as mean \pm SD for at least triplicate determination, and analyzed using Fisher's exact test and Kruskal-Wallis test. All statistical analyses were performed using SPSS 13.0 and $\mathrm{P}<0.05$ was considered as statistically significant.

\section{Results}

\section{NSBP1 expression is high in ccRCC tissues}

We examined NSBP1 expression in ccRCC tissue by immunohistochemistry. As shown in Figure 1A, NSBP1 staining was weak in the normal renal tissues but strong in ccRCC tissues. Western blot analysis of 20 paired adjacent normal renal tissues and ccRCC tissues confirmed the high expression of NSBP1 in ccRCC tissues $(\mathrm{p}=0.006)$ (Figure 1B). Most importantly, we found that NSBP1 staining intensity was correlated with the clinical and pathologic characteristics of ccRCC (Table 1). NSBP1 expression was positively correlated with the tumor grade and pathologic stage. 
A.

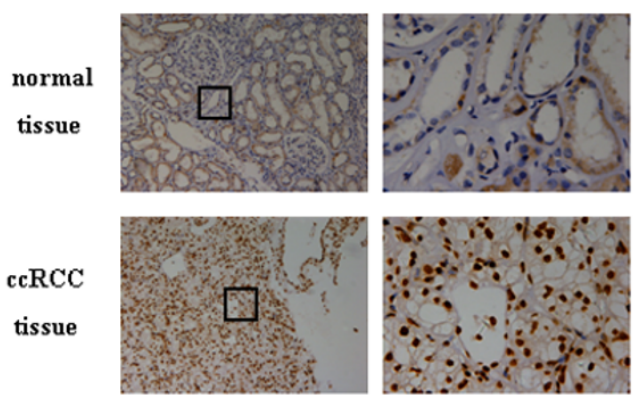

B.

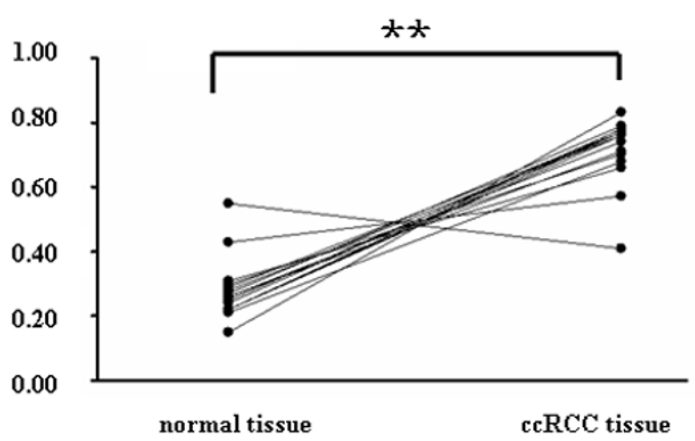

C.

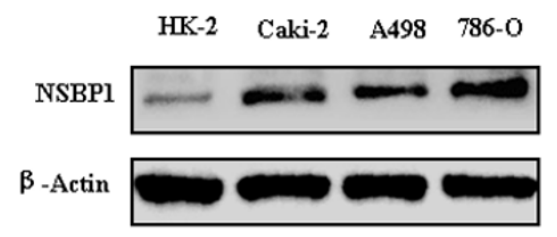

D.

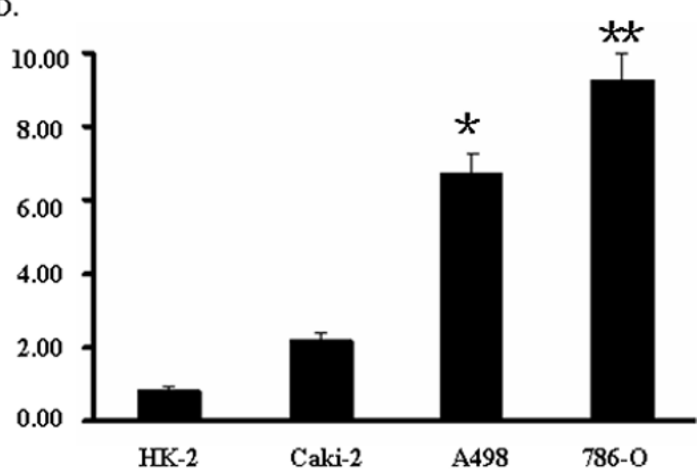

Figure 1 NSBP1 expression is high in ccRCC tissues and cells. (A), Representative immunohistochemistry staining of NSBP1 in control renal tissue and ccRCC tissue. NSBP1 immunoreactivity in brown was predominantly localized in the nucleus. Original magnification: X10 ( $a, c), X 40$ (b, d). (B) Ratio between protein expression levels of NSBP1 and $\beta$-Actin in pairs of ccRCC and normal tissue from 20 patients was calculated based on Western blot analysis. (C), Western blots demonstrating the expression of NSBP1 in different CcRCC cells. Actin served as loading control. (D), Real-time PCR assay showing the relative NSBP1 mRNA level in different $c C R C C$ cells. ${ }^{*} p<0.05,{ }^{* *} p<0.01$, versus HK-2 cells.

Table 1 Correlation of NSBP1 expression with clinical and pathological characteristics of renal carcinoma

\begin{tabular}{|c|c|c|c|c|c|c|c|c|}
\hline \multirow[t]{3}{*}{ Characteristics } & \multirow[t]{3}{*}{ Cases } & \multicolumn{6}{|c|}{ NSBP1 immunoreactivity } & \multirow[t]{3}{*}{$P$} \\
\hline & & & - & & + & & ++ & \\
\hline & & \multicolumn{2}{|c|}{$\begin{array}{c}\text { Cases } \\
(\%)\end{array}$} & \multicolumn{2}{|c|}{$\begin{array}{c}\text { Cases } \\
(\%)\end{array}$} & \multicolumn{2}{|c|}{$\begin{array}{c}\text { Cases } \\
(\%)\end{array}$} & \\
\hline Gender & & & & & & & & 0.653 \\
\hline Male & 129 & 18 & 11.8 & 33 & 21.7 & 78 & 51.3 & \\
\hline Female & 23 & 3 & 2.0 & 8 & 5.3 & 12 & 7.9 & \\
\hline Age (years) & $60.4 \pm 8.9$ & & $\begin{array}{l}9.8 \pm \\
9.7\end{array}$ & & $\begin{array}{l}0.2 \pm \\
9.8\end{array}$ & 61.3 & $3 \pm 11$ & \\
\hline Grade & & & & & & & & $0.040^{*}$ \\
\hline 1 & 0 & 0 & & 0 & & 0 & & \\
\hline 2 & 63 & 14 & 9.2 & 16 & 10.5 & 33 & 21.7 & \\
\hline 3 & 89 & 7 & 4.6 & 25 & 16.5 & 57 & 37.5 & \\
\hline Pathologic stage & & & & & & & & $0.002^{* *}$ \\
\hline $\mathrm{T} 1$ & 18 & 10 & 6.6 & 5 & 3.3 & 3 & 2.0 & \\
\hline $\mathrm{T} 2$ & 35 & 6 & 3.9 & 12 & 7.9 & 17 & 11.1 & \\
\hline Т3 & 47 & 3 & 2.0 & 15 & 9.9 & 29 & 19.1 & \\
\hline T4 & 52 & 2 & 1.3 & 9 & 5.9 & 41 & 27.0 & \\
\hline
\end{tabular}

Correlation significant at the 0.05 level (one-tailed)

${ }^{*} \mathrm{P}<0.05 ;{ }^{* * P}<0.01$
NSBP1 expression is high in ccRCC cells

We examined NSBP1 expression in ccRCC cell lines and the normal renal tubular epithelial line cells by quantitative real-time RT-PCR and Western blot. NSBP1 protein level was higher in ccRCC cell lines than normal renal tubular epithelial line cells (Figure 1C). Similarly, NSBP1 mRNA level was increased in ccRCC cell lines compared to normal renal tubular epithelial line cells (Figure 1D).

\section{NSBP1 knockdown decreases the proliferation of ccRCC cells}

To investigate the role of NSBP1 in the proliferation of ccRCC cells, we employed the loss of function approach. 786-O cells were transfected with NSBP1 siRNA or scramble siRNA as control and cell proliferation was evaluated by MTT assay. The results showed that NSBP1 knockdown significantly reduced proliferation of ccRCC cells over the $72 \mathrm{~h}$ period (Figure 2A). Lentivirus short hairpin constructs against NSBP1 (PscSI616) was efficient and specific in the knockdown of NSBP1 in 786-O cells and the inhibitory efficiency at protein level was $74.8 \pm$ 2.1\% based on Western blot analysis (Figure 2C). 


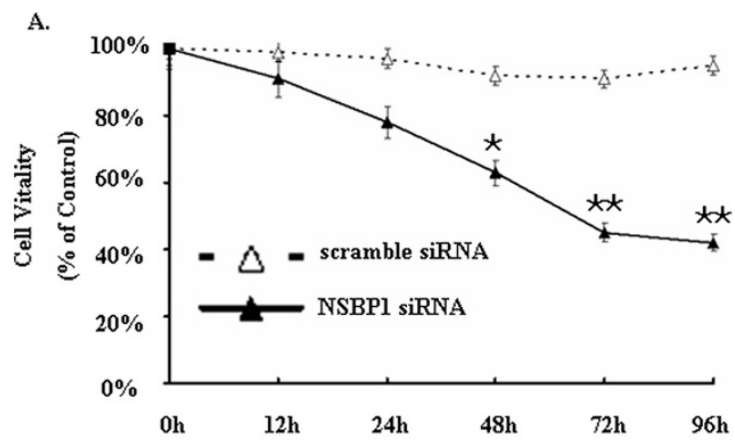

B.

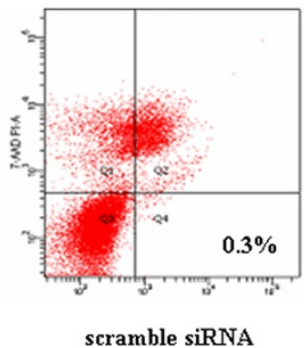

C.

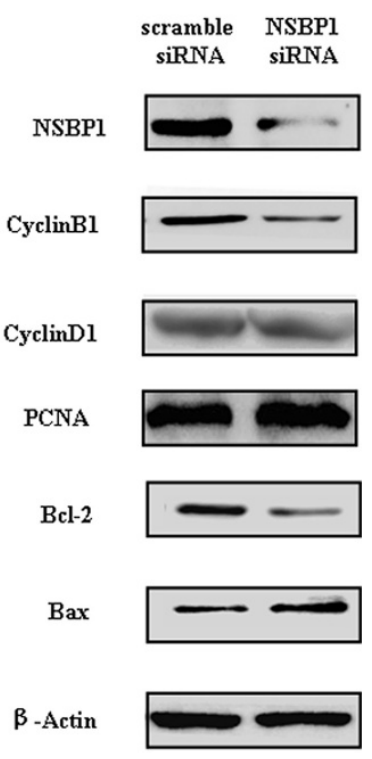

Figure 2 NSBP1 knockdown decreases the proliferation of ccRCC cells. (A), MTT assay showing that NSBP1 knockdown significantly reduced the proliferation of cCRCC cells over the $96 \mathrm{~h}$ period. (B), Annexin V-PE/7-AAD staining in FCM assays showing the ratio of apoptosis in different 786-O cells. (Data were presented as mean \pm SEM, $n=3$ ). (C), Representative blots demonstrating the reduced protein level of NSBP1 in NSBP1 siRNA transfected 786-O cells compared to scramble siRNA transfected cells. In addition, Bax protein level was increased and CyclinB1 and Bcl-2 levels were decreased in NSBP1 siRNA transfected 786-O cells compared to scramble siRNA transfected cells.

The apoptosis of ccRCC cells was examined by FCM after the cells were transfected with NSBP1 siRNA or scramble siRNA as control. The apoptotic ratio was increased in NSBP1 knockdown 786-O cells compared to control (Figure 2B). To confirm that NSBP1 knockdown could inhibit proliferation and induce apoptosis in ccRCC cells, we examined the expression of apoptosis and cell cycle related proteins and found that Bax protein level was significantly increased while CyclinB1 and Bcl-2 protein levels were decreased in NSBP1 knockdown cells compared with control (Figure 2C). These data provide evidence that NSBP1 modulates cell cycle and antagonizes apoptosis to promote the oncogenic potential of ccRCC cells.

\section{NSBP1 knockdown inhibits the invasion of ccRCC cells}

Next we assessed the role of NSBP1 in cell invasion, an important aspect of ccRCC metastasis. By transwell assay we found that NSBP1 knockdown cells showed few number of invading cells compared to control group which expressed high level of NSBP1 (Figure 3A). The number of cells crossing the matrigel was $62.3 \pm$ 3.1 in NSBP1 siRNA group versus $110.7 \pm 3.1$ in scramble siRNA control group $(\mathrm{P}<0.05)$. Moreover, gelatin zymography assay demonstrated that NSBP1 knockdown efficiently decreased MMP-2 and MMP-9 enzymatic activity, especially MMP-9 enzymatic activity (Figure 3B). To address whether decreased MMP-9 and MMP-2 activity is due to the downreguation of their expression after NSBP1 knockdown, we examined the expression of MMP-9, MMP-2 and their upstream transcription factors c-fos and c-jun. Western blot analysis demonstrated that NSBP1 knockdown downregulated the expression of VEGF, VEGFR-2, MMP-2, MMP-9, c-fos and c-jun (Figure $3 C$ ). Taken together, these data suggest that NSBP1 upregulates the expression of MMP-2 and MMP-9 via c-fos and c-jun. The increased MMPs activity and angiogenesis then contributes to the migration and invasion of ccRCC cells.

\section{NSBP1 knockdown inhibits ccRCC growth in xenograft nude mice}

To further investigate the role of NSBP1 in ccRCC in vivo, we established xenograft ccRCC by subcutaneous injection of $1 \times 10^{6}$ NSBP1 knockdown 786-O cells or the corresponding scramble siRNA transfected control cells into the flanks of BALB/c nude mice $(n=10)$. After 6 weeks, we observed that the volume of tumor derived from NSBP1 knockdown cells was significantly smaller than that derived from control cells (Figure 4). These data demonstrate that NSBP1 knockdown inhibits the tumorigenicity of ccRCC cells in vivo. 


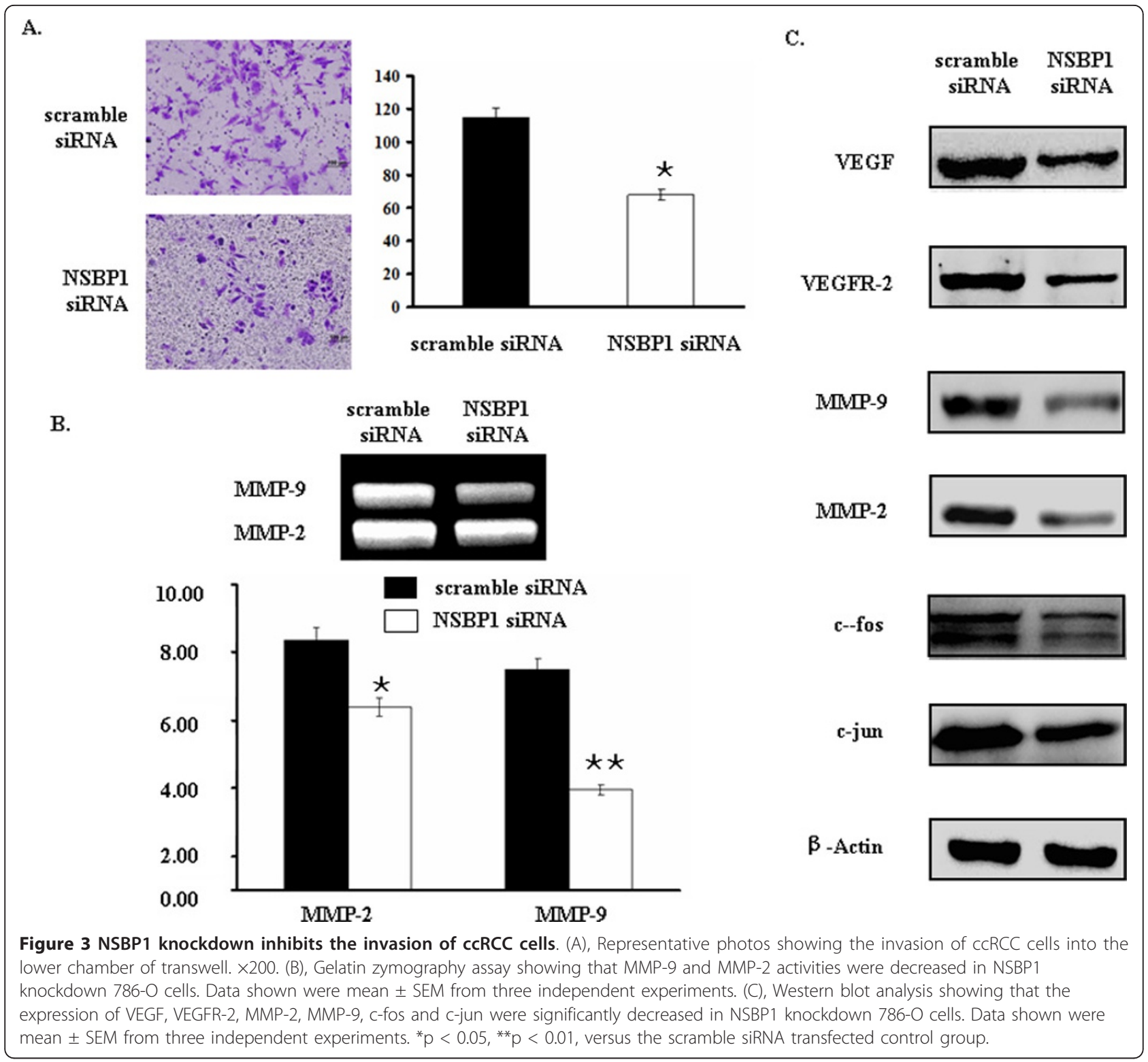

\section{Discussion}

NSBP1 was identified as a new member of the HMGN protein family in 2001 [12,13]. As a nuclear protein, NSBP1 modulates the structure and function of chromatin and plays an important role in transcription, DNA replication and repair [14-16]. Interestingly, recent studies demonstrated that NSBP1 expression was abnormally high in a variety of solid tumors, indicating the oncogenic role of NSBP1 [4-7],

In this study, we found that NSBP1 expression was significantly higher in ccRCC tissues and cell lines than normal renal tissue and cell lines. These data suggest that NSBP1 overexpression is correlated with the progression of ccRCC.
To elucidate the role of NSBP1 in the tumorigenesis of ccRCC, we employed loss of function approach via the knockdown of endogenous NSBP1 expression in ccRCC cells. Notably, we found that NSBP1 knockdown inhibited the proliferation rate of ccRCC cells through MTT assay. Furthermore, our experiments showed that knockdown of NSBP1 led to increased Bax expression and decreased CyclinB1, Bcl-2 expression. These results suggest that downregulation of NSBP1 expression causeds G2 cell cycle arrest, decreases the proliferation rate and increases apoptosis rate in ccRCC cells in vitro[17-20].

Metastasis is an important aspect of ccRCC. To characterize the role of NSBP1 in ccRCC metastasis, we employed in vitro invasion assay and found that NSBP1 
A.

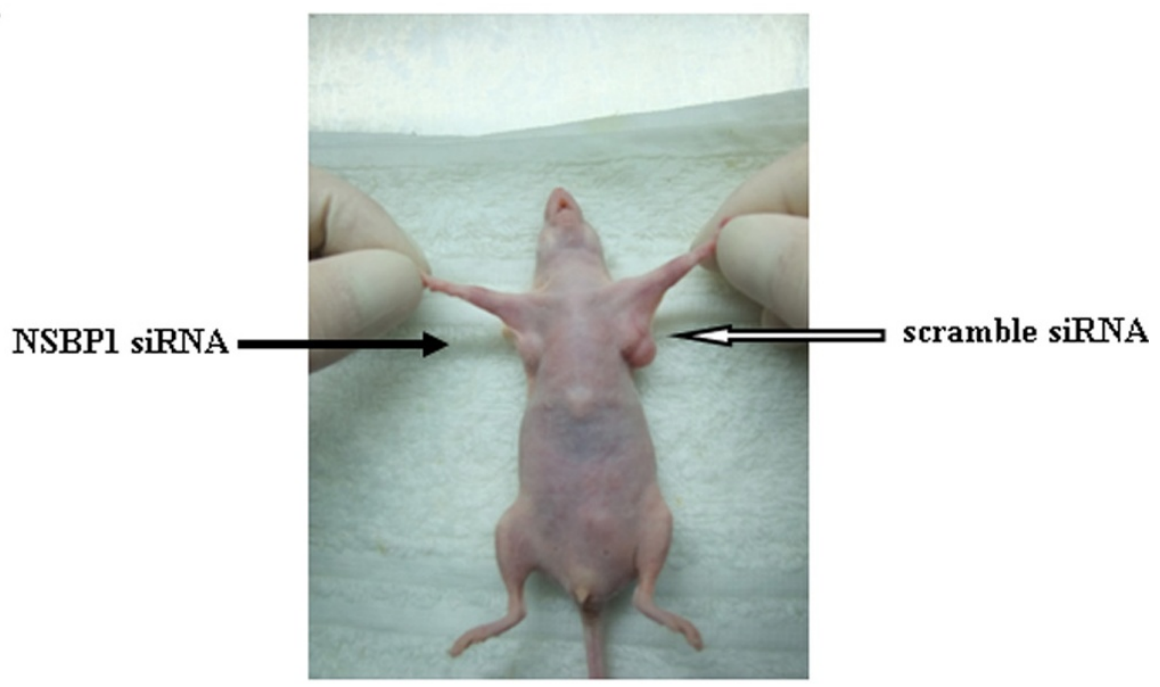

B.

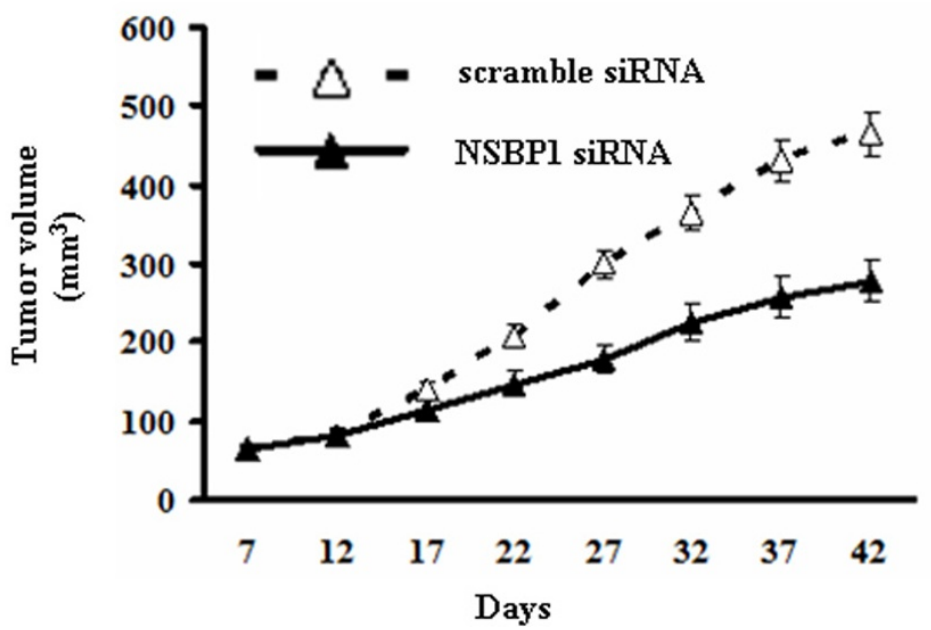

Figure 4 NSBP1 knockdown inhibits the tumorigenicity of ccRCC cells in vivo. (A), A representative nude mice showing the different morphology of the tumors derived from NSBP1 siRNA transfected 786-O cells (left side) and scramble siRNA transfected control cells (right side). (B), the growth curve of the tumors $(n=10)$.

knockdown led to decreased invasion of ccRCC cells. Tumor invasion and metastasis are crucially dependent on MMPs and VEGF $[10,11,20]$. MMP-2 and MMP-9 play important roles in the degradation of the ECM, including type IV collagen, and their activity and expression are correlated with metastatic abilities and prognosis of cancer $[21,22]$. Our results showed that silencing of NSBP1 in 786-O cells decreased MMP- 2 and MMP-9 activity based on zymography assay. In addition, we found that MMP-2 and MMP-9 expression as well as the expression of transcription factors c-fos and c-jun were decreased in NSBP1 knockdown cells. These data suggest that NSBP1 modulates the expression of MMPs and VEGF/VEGFR-2 thus influencing the invasion behavior of ccRCC cells.
Finally, to demonstrate that NSBP1 contributes to ccRCC development in vivo, we employed xenograft nude mice model and found that NSBP1 knockdown suppressed tumor growth of ccRCC cells, indicating that NSBP1 promotes the tumorigenicity of ccRCC cells in vivo.

In summary, here we present both in vitro and in vivo evidence that NSBP1 promotes ccRCC cells growth and invasion. NSBP1 plays important role in the regulation of apoptosis and invasion of ccRCC cells by regulating the expression of Bcl-2, Bax, CyclinB1 VEGF/VEGFR-2 and MMPs. Based on these findings, intervention with NSBP1 expression may provide a therapeutic approach in ccRCC development and metastasis. 


\section{Acknowledgements}

The work was supported by grants from the National Natural Science Foundation of China (No.30271295 and 30672099) and Beijing Natural Science Foundation (No.7092101).

\section{Author details}

'Department of Urology, Peking University First Hospital, Beijing 100034, China. ${ }^{2}$ The Institute of Urology, Peking University, Beijing 100034, China. ${ }^{3}$ National Urological Cancer Center, Beijing 100034, China. ${ }^{4}$ Department of Urology, First Hospital of Peking University, Peking University, No. 8, Xishiku Street, West District, Beijing 100034, China.

\section{Authors' contributions}

SQJ supervised research project, participated in the data collection, drafted the manuscript. LY participated in the data collection, supervised ICH. XYZ participated in the data collection. XSL carried out the operation. LQZ carried out the operation, acted as corresponding author and did the revisions. All authors read and approved the final manuscript.

\section{Competing interests}

The authors declare that they have no competing interests.

Received: 9 February 2012 Accepted: 15 March 2012

Published: 15 March 2012

\section{References}

1. Ljungberg B, Campbell SC, Choi HY, Jacqmin D, Lee JE, Weikert S, Kiemeney LA: The epidemiology of renal cell carcinoma. Eur Urol 2011, 60:615-621.

2. Hock R, Furusawa T, Ueda T, Bustin M: HMG chromosomal proteins in development and disease. Trends Cell Biol 2007, 17:72-79.

3. Wang JW, Zhou LQ, Yang XZ, Ai JK, Xin DQ, Na YQ, Guo YL: The NSBP1 expression is up-regulated in prostate cancer cell. Basic Med Sci Clin 2004, 24:393-397.

4. Huang C, Zhou LQ, Song G: Effect of nucleosomal binding protein 1 in androgen-independent prostatic carcinoma. Zhong hua Yi Xue Za Zhi 2008, 88:657-660.

5. Green J, Ikram M, Vyas J, Patel N, Proby CM, Ghali L, Leigh IM, O'Toole EA Storey A: Overexpression of the Axl tyrosine kinase receptor in cutaneous SCC-derived cell lines and tumours. Br J Cancer 2006, 94:1446-1451.

6. Li DQ, Hou YF, Wu J, Chen Y, Lu JS, Di GH, Ou ZL, Shen ZZ, Ding J, Shao ZM: Gene expression profile analysis of an isogenic tumour metastasis model reveals a functional role for oncogene AF1Q in breast cancer metastasis. Eur J Cancer 2006, 42:3274-3286.

7. Tang WY, Newbold R, Mardilovich K, Jefferson W, Cheng RY, Medvedovic M, Ho SM: Persistent hypomethylation in the promoter of nucleosomal binding protein 1 (Nsbp1) correlates with overexpression of Nsbp1 in mouse uteri neonatally exposed to diethylstilbestrol or genistein. Endocrinology 2008, 149:5922-5931.

8. Zhou LQ, Song G, He ZS, Hao JR, Na YQ: Effect of inhibiting nucleosomal binding protein 1 on proliferation of human prostate cancer cell line LNCaP. Chin Med J 2007, 86:404-408.

9. Jiang $N$, Zhou $L Q$, Zhang $X Y$ : Downregulation of the nucleosome-binding protein 1 (NSBP1) gene can inhibit the in vitro and in vivo proliferation of prostate cancer cells. Asian J Androl 2010, 12:709-717.

10. Mukherjee S, Roth MJ, Dawsey SM, Yan W, Rodriquez-Canales J, Erickson HS, Hu N, Goldstein AM, Taylor PR, Richardson AM, Tangrea MA, Chuaqui RF, Emmert-Buck MR: Increased matrix metalloproteinase activation in esophageal squamous cell carcinoma. J Transl Med 2010, 8:91.

11. Rak J, Milsom C, May L, Klement P, Yu J: Tissue factor in cancer and angiogenesis: the molecular link between genetic tumor progression tumor neovascularization, and cancer coagulopathy. Semin Thromb Hemost 2006, 32:54-70, Review.

12. Rochman M, Malicet C, Bustin M: HMGN5/NSBP1: A new member of the HMGN protein family that affects chromatin structure and function. Biochim Biophys Acta 2010, 1799:86-92.

13. Shirakawa H, Herrera JE, Bustin M, Postnikov Y: Targeting of high mobility group-14/-17 proteins in chromatin is independent of DNA sequence. $J$ Biol Chem 2000, 275:37937-37944.
14. Catez F, Lim JH, Hock R, Postnikov YV, Bustin M: HMGN dynamics and chromatin function. Biochem Cell Biol 2003, 81:113-122.

15. Rochman M, Postnikov Y, Correll S, Malicet C, Wincovitch S, Karpova TS, McNally JG, Wu X, Bubunenko NA, Grigoryev S, Bustin M: The interaction of NSBP1/HMGN5 with nucleosomes in euchromatin counteracts linker histone-mediated chromatin compaction and modulates transcription, Mol. Cell 2009, 35:642-656.

16. Rattner BP, Yusufzai T, Kadonaga JT: HMGN proteins act in opposition to ATP-dependent chromatin remodeling factors to restrict nucleosome mobility. Mol Cell 2009, 34:620-626.

17. Rozenblat S, Grossman S, Bergman M, Gottlieb H, Cohen Y, Dovrat S: Induction of G2/M arrest and apoptosis by sesquiterpene lactones in human melanoma cell lines. Biochem Pharmacol 2008, 75:369-382.

18. Beauman SR, Campos B, Kaetzel MA, Dedmana JR: CyclinB1 expression is elevated and mitosis is delayed in HeLa cells expressing autonomous CaMKII. Cell Signal 2003, 15:1049-1057.

19. Chulu LCJulius, Huang Wei R, Wang L, Shih Wen L, Liu Hung J: Avian Reovirus Nonstructural Protein p17-Induced G2/M Cell Cycle Arrest and Host Cellular Protein Translation Shutoff Involve Activation of p53Dependent Pathways. J Virol 2010, 84:7683-7694.

20. Yin J, Chen G, Liu Y, Liu S, Wang P, Wan Y, Wang X, Zhu J, Gao H: Downregulation of SPARC expression decreases gastric cancer cellular invasion and survival. J Exp Clin Cancer Res 2010, 29:59.

21. Rink M, Chun FK, Robinson B, Sun M, Karakiewicz PI, Bensalah K, Fisch M, Scherr DS, Lee RK, Margulis V, Shariat SF: Tissue-based molecular markers for renal cell carcinoma. Minerva Urol Nefrol 2011, 63:293-308.

22. Chang HR, Chen PN, Yang SF, Sun YS, Wu SW, Hung TW, Lian JD, Chu SC, Hsieh YS: Silibinin inhibits the invasion and migration of renal carcinoma 786-O cells in vitro, inhibits the growth of xenografts in vivo and enhances chemosensitivity to 5-fluorouracil and paclitaxel. Mol Carcinog 2011, 50:811-823.

doi:10.1186/1756-9966-31-22

Cite this article as: Ji et al:: Knockdown of the nucleosome binding protein 1 inhibits the growth and invasion of clear cell renal cell carcinoma cells in vitro and in vivo. Journal of Experimental \& Clinical Cancer Research 2012 31:22.

\section{Submit your next manuscript to BioMed Central and take full advantage of:}

- Convenient online submission

- Thorough peer review

- No space constraints or color figure charges

- Immediate publication on acceptance

- Inclusion in PubMed, CAS, Scopus and Google Scholar

- Research which is freely available for redistribution

Submit your manuscript at www.biomedcentral.com/submit
C Biomed Central 